\title{
Publisher Correction: Concerns with yeast mitochondrial ADP/ATP carrier's integrity in DPC
}

Martin S. King, Paul G. Crichton (D), Jonathan J. Ruprecht and Edmund R. S. Kunji

Correction to: Nature Structural \& Molecular Biology https://doi.org/10.1038/s41594-018-0125-6, published online 3 September 2018. In the version of this article originally published, references 6 and 7 were interchanged in the reference list. The error has been corrected in the HTML and PDF versions of the article.

Published online: 14 September 2018

https://doi.org/10.1038/s41594-018-0138-1 